\title{
Research on personality characteristics and presentation characteristics of Elite Archers
}

\author{
Lin jia huan ${ }^{1, a}$ \\ China; Zhejiang; 9 Xuefu Road, Yinzhou District, Ningbo (Ningbo College of Vocational \\ Technology)
}

Keywords: excellent, archers; personality characteristics; sports imagery.

\begin{abstract}
PF personality characteristics and SIQ movement appearance of excellent archers, the literature, experimental and mathematical statistics were used to investigate and analyze. The results show that excellent athletes are good at dealing with interpersonal relationship, strong ability to adapt to the environment, mature and stable mood, self-discipline and prudence, self-discipline, self confidence, the imagery of stimulating the control motivation and the better ability of arousing arousal motivation, and select nine factors that affect the achievement of excellent archery mobilization and carry out regression points. There were significant differences in Q4 tension, O anxiety, L skepticism, Q2 independence, I sensitivity and B intelligence.
\end{abstract}

\section{优秀射箭运动员人格特征及表象特点研究}

\author{
林甲换 ${ }^{1, a}$ \\ 1宁波城市职业技术学院, 鄞州, 宁波, 浙江, 中国
}

关键词:优秀射箭运动员; 人格特征; 运动表象

中文摘要: 为了探讨优秀射箭运动员 $16 \mathrm{PF}$ 人格特征及 SIQ 运动表象特点, 运用文献资料 法、实验研究法和数理统计法, 进行调查分析。结果显示: 优秀运动员善于处理人际关系, 适应环境的能力较强, 情绪的成熟稳定,自律谨慎, 沉着, 有自信心, 激发控制动机的表象与 激发唤醒动机的表象训练能力较好; 选取影响优秀射箭运动员成绩的九个因子,并进行回归分 析,其中具有显著性差异的因素为 Q4 紧张性, $\mathrm{O}$ 忧虑性, $\mathrm{L}$ 怀疑性, Q2 独立性、I 每感性 和 B 聪慧性。

\section{1. 引言}

射箭运动属于间接竞技类准确性项目，其竞技能力的核心是准确性和稳定性。其稳定性 除了要求运动员在动作过程中具有良好的上肢本体感觉外, 还和运动员自身心理特质如人格 具有必然联系。在运动员习得的运动技能中, 心理技能在竞赛中也会发挥举足轻重的作用, 一名优秀运动员在表象过程中，其肌电特征跟训练过程中所诱发肌电相差无几，这充分说明 了表象的重要性。

\section{2. 研究对象和方法}

\section{1研究对象}


选取上海、山东、福建三省优秀射箭运动员 54 名作为研究对象, 全部为男性。被试者年 龄为 $19 \sim 25$ 岁 $(21.37 \pm 2.21)$, 训练年限为2 $5(3.360 \pm 1.567)$ 年, 均参加过射箭比赛且获得 过优异的成绩。

\section{2研究方法}

2.2.1文献资料法：查阅射箭相关文献，了解相关研究现状。

2.2.2 问卷调查法: 搜集2012年优秀运动员共54名。同时对这些运动员进行 $16 \mathrm{PF}$ 和SIQ量表的 问卷调查。

\subsection{3数理统计法}

本文对射箭运动员 $16 \mathrm{PF}$ 常模指标、运动表象指标进行描述, 将射箭成绩设为因变量, 各 心理指标设为自变量, 通过因子分析法, 找出影响较大的几个主要成分, 再将主成分因子进 行线性回归分析。

\section{3. 结果与分析}

\section{1 优秀射箭运动员 $16 P F$ 常模的标定}

根据卡特尔（R.B. Cattell）所提出的标准分 $5.5 \pm 2$ 及相应的概率,标定优秀射箭运动员常 模, 将 16 个人格因素做描述统计，如表 1 所示。

表 1 人格标准分数及其相应的百分率一览表

\begin{tabular}{lcccccccccc}
\hline $\begin{array}{l}\text { 标准 } \\
\text { 分 }\end{array}$ & 1 & 2 & 3 & 4 & 5 & 6 & 7 & 8 & 9 & 10 \\
\hline $\begin{array}{l}\text { 常态 } \\
\text { 分布 }\end{array}$ & 1.4 & 2.5 & 8.0 & 12.5 & 24.0 & 24.8 & 13.0 & 9.1 & 3.3 & 1.4 \\
$\begin{array}{c}\text { 累加 } \\
\text { 比例 }\end{array}$ & 1.4 & 3.9 & 11.9 & 24.4 & 48.5 & 73.2 & 86.2 & 95.3 & 98.6 & 100 \\
\hline
\end{tabular}

\section{2 优秀射箭运动员常模}

\begin{tabular}{|c|c|c|}
\hline \multirow[t]{2}{*}{ 因素 } & \multicolumn{2}{|c|}{ 射箭运动员（ $\mathrm{N}=54$ 人） } \\
\hline & M & $\mathrm{SD}$ \\
\hline A(乐群) & 10.88 & 2.75 \\
\hline B(聪慧) & 8.04 & 1.82 \\
\hline $\mathrm{C}$ (稳定) & 16.90 & 3.60 \\
\hline E（恃强性） & 11.13 & 3.01 \\
\hline F(兴奋) & 14.04 & 3.40 \\
\hline G(有恒) & 12.77 & 2.97 \\
\hline $\mathrm{H}$ (敢为) & 12.75 & 3.43 \\
\hline I(敏感) & 11.36 & 2.58 \\
\hline $\mathrm{L}$ (怀疑） & 7.94 & 2.52 \\
\hline $\mathrm{M}($ 幻想 $)$ & 11.90 & 3.02 \\
\hline $\mathrm{N}$ (世故） & 8.86 & 2.46 \\
\hline $\mathrm{O}$ (忧虑) & 8.92 & 3.65 \\
\hline Q1(实验) & 10.13 & 2.58 \\
\hline Q2(独立) & 10.96 & 2.81 \\
\hline Q3(自律) & 12.87 & 2.68 \\
\hline Q4(紧张) & 11.19 & 3.48 \\
\hline
\end{tabular}

由表 2 所示, 16 种人格中, 优秀射箭运动员出现高 $\mathrm{A}($ 乐群)、高 $\mathrm{C}($ 稳定)、高 $\mathrm{F}$ (兴 奋)、高 $\mathrm{H}$ (敢为) 、高 $\mathrm{Q} 3$ (自律)且低 $\mathrm{G}$ (有恒)、低 $\mathrm{L}($ 怀疑 $)$ 、低 $\mathrm{M}($ 幻想 $)$ 、低 $\mathrm{O}($ 忧虑 $)$ 、低 
$\mathrm{Q} 1($ 实验) 、低 $\mathrm{Q} 2($ 独立) 、低 $\mathrm{Q} 4($ (紧张)，结果表明：优秀射箭运动员善于处理人际关 系, 适应环境的能力较强, 情绪的成熟稳定、自律谨慎, 沉着, 有自信心, 但不爱幻想, 创 造能力较低, 思维比较墨守成规, 缺乏独立能力, 依赖能力较强。

\section{3 优秀射箭运动员运动表象情况}

\begin{tabular}{|c|c|c|c|c|c|}
\hline & $\begin{array}{l}\text { 激发特殊动机的 } \\
\text { 表象 }\end{array}$ & $\begin{array}{l}\text { 激发唤醒动机的 } \\
\text { 表象 }\end{array}$ & $\begin{array}{l}\text { 激发控制动机的 } \\
\text { 表象 }\end{array}$ & $\begin{array}{l}\text { 特殊认知的表 } \\
\text { 象 }\end{array}$ & $\begin{array}{l}\text { 一般认知的表 } \\
\text { 象 }\end{array}$ \\
\hline 平均 & $21.00 \pm 9.45$ & $23.02 \pm 6.37$ & $28.62 \pm 6.32$ & $28.28 \pm 7.47$ & $25.78 \pm 7.28$ \\
\hline
\end{tabular}

如表 3 所示，优秀射箭运动员运动表象中，激发控制动机的表象的均值在各维度中居最 高为 28.62 , 居于其次是特殊认知表象分值为 28.28 , 激发特殊动机的表象与一般认知的表象 的均值较低。结果表明: 在平时训练过程中, 知觉运动技能方面的表象训练比较多, 目标设 置的相关联表象能力其次, 而控制自己的躯体和情绪的表象能力较弱, 在对某一种竞技情景 中的策略做出表象性的表述能力不强, 需在今后加强激发特殊动机表象和激发唤醒动机的表 象训练。

\section{4 优秀射箭运动员心理指标的因子提取检验}

表 4 优秀射箭运动员心理指标主成分提取的适合程度检验

\begin{tabular}{lrr}
\hline \multicolumn{2}{c}{ 取样足够度的 Kaiser-Meyer-Olkin 变量 } & .659 \\
\hline Bartlett 的球形度检验 & 近拟卡方 & 254.206 \\
& df & 190 \\
& Sig. & .001 \\
\hline
\end{tabular}

将优秀射箭运动员各个心理指标进行因子提取的适合度检验 (MO 和 Bartlett 的检 验), 来判断所获得的数据是否能建立因子模型。如表 4 所示: 心理因素的 KMO 数值为 0.659 , Bartlett 球形检验的进拟卡方值为 $254.206, \mathrm{P}<0.000$ 可以进行因子分析。

\section{5 优秀射箭运动员心理指标主成分的提取}

表 5 心理因素主成分提取的因子共同度

\begin{tabular}{ccc}
\hline 因素 & 出始 & 提取 \\
\hline $\mathrm{A}$ 乐群 & 1.000 & .810 \\
$\mathrm{~B}$ 聪慧 & 1.000 & .722 \\
$\mathrm{C}$ 稳定 & 1.000 & .758 \\
$\mathrm{E}$ 待强 & 1.000 & .627 \\
$\mathrm{~F}$ 兴奋 & 1.000 & .674 \\
$\mathrm{G}$ 永恒 & 1.000 & .823 \\
$\mathrm{H}$ 敢为 & 1.000 & .723 \\
$\mathrm{I}$ 敏感 & 1.000 & .528 \\
$\mathrm{~L}$ 怀疑 & 1.000 & .820 \\
$\mathrm{M}$ 幻想 & 1.000 & .838 \\
$\mathrm{~N}$ 事故 & 1.000 & .688 \\
$\mathrm{O}$ 忧虑 & 1.000 & .655 \\
$\mathrm{Q} 1$ 实验 & 1.000 & .627 \\
$\mathrm{Q} 2$ 独立 & 1.000 & .761 \\
$\mathrm{Q} 3$ 自律 & 1.000 & .676 \\
$\mathrm{Q} 4$ 紧张 & 1.000 & .656 \\
一般认知的表象 & 1.000 & .775 \\
特殊认知的表象 & 1.000 & .856 \\
激发控制动机的表象 & 1.000 & .835 \\
激发唤醒动机的表象 & 1.000 & .736 \\
\hline
\end{tabular}


由表 5 得出: 在变量共同度为 1 的情况下（将变量以 1 为方差将其标准化）, 除 $\mathrm{B}$ 聪 慧、 $\mathrm{E}$ 待强、 $\mathrm{F}$ 兴奋等 11 个因素外, 变量的部分信息都可以较好地被因子解释(共同度> $0.75)$, 表明提取的因子能够保证使变量信息不被丢失。

\section{6 优秀射箭运动员主成分个数因子的确定}

表 6 优秀射箭运动员各指标因子分析的总方差解释

\begin{tabular}{|c|c|c|c|c|c|c|c|c|c|}
\hline \multirow[t]{2}{*}{ 成分 } & \multicolumn{3}{|c|}{ 初始特征值 } & \multicolumn{3}{|c|}{ 提取平方和载入 } & \multicolumn{3}{|c|}{ 旋转平方和转入 } \\
\hline & 合计 & 方差的\% & 累计 \% & 合计 & $\begin{array}{l}\text { 方差 } \\
\text { 的\% }\end{array}$ & 累计\% & 合计 & 方差\% & 累积 $\%$ \\
\hline 1 & 3.482 & 17.409 & 17.409 & 3.482 & 17.409 & 17.409 & 3.231 & 16.156 & 16.156 \\
\hline 2 & 2.381 & 11.903 & 29.312 & 2.381 & 11.903 & 29.312 & 2.029 & 10.145 & 26.302 \\
\hline 3 & 1.775 & 8.876 & 38.188 & 1.775 & 8.876 & 38.188 & 1.717 & 8.585 & 34.887 \\
\hline 4 & 1.429 & 7.144 & 45.333 & 1.429 & 7.144 & 45.333 & 1.569 & 7.845 & 42.732 \\
\hline 5 & 1.260 & 6.299 & 51.632 & 1.260 & 6.299 & 51.632 & 1.318 & 6.588 & 49.320 \\
\hline 6 & 1.232 & 6.159 & 57.791 & 1.232 & 6.159 & 57.791 & 1.223 & 6.117 & 55.437 \\
\hline 7 & 1.149 & 5.744 & 63.535 & 1.149 & 5.744 & 63.535 & 1.188 & 5.941 & 61.378 \\
\hline 8 & .980 & 4.899 & 68.434 & .980 & 4.899 & 68.434 & 1.171 & 5.854 & 67.233 \\
\hline 9 & .900 & 4.501 & 72.935 & .900 & 4.501 & 72.935 & 1.140 & 5.702 & 72.935 \\
\hline 10 & .830 & 4.150 & 77.084 & & & & & & \\
\hline 11 & .726 & 3.629 & 80.713 & & & & & & \\
\hline 12 & .675 & 3.373 & 84.086 & & & & & & \\
\hline 13 & .608 & 3.040 & 87.126 & & & & & & \\
\hline 14 & .565 & 2.827 & 89.953 & & & & & & \\
\hline 15 & .531 & 2.655 & 92.608 & & & & & & \\
\hline
\end{tabular}

由因子反映整体各变量的信息情况看, 确定了选择提取 9 个因子为较佳方案。如表 6 所 示，9 个因子可以解释原有变差的 72.94\%（方差累计贡献率 $>72 \%$ ）。

\section{7 优秀射箭运动员指标的因子总分计算函数的建立。}

\begin{tabular}{|c|c|c|c|c|c|c|c|c|c|}
\hline & \multicolumn{9}{|c|}{ 成份 } \\
\hline & F1 & F2 & F3 & F4 & F5 & F6 & F7 & F8 & F9 \\
\hline B 聪慧 & .069 & -.228 & .175 & .659 & .186 & -.334 & -.129 & -.274 & -.225 \\
\hline $\mathrm{C}$ 稳定 & .061 & -.374 & .601 & -.022 & .102 & .374 & -.182 & .075 & -.166 \\
\hline $\mathrm{E}$ 待强 & .104 & -.037 & -.108 & .075 & .072 & .839 & -.041 & -.067 & .115 \\
\hline $\mathrm{F}$ 兴奋 & .203 & .109 & .109 & .566 & -.202 & .287 & .063 & .252 & .225 \\
\hline$G$ 永恒 & -.041 & -.080 & .689 & .271 & -.166 & -.136 & -.205 & .112 & .129 \\
\hline $\mathrm{H}$ 敢为 & .071 & -.127 & .088 & .099 & -.005 & .121 & .058 & -.072 & .872 \\
\hline I 敏感 & .073 & .322 & .214 & .114 & .532 & -.144 & -.068 & .409 & .283 \\
\hline $\mathrm{L}$ 怀疑 & .157 & .670 & -.054 & -.020 & -.044 & -.038 & -.136 & -.099 & .139 \\
\hline M 幻想 & .053 & -.086 & -.050 & .063 & .047 & -.057 & .891 & -.033 & .061 \\
\hline $\mathrm{N}$ 事故 & .063 & -.183 & -.020 & .120 & .006 & -.039 & -.027 & .879 & -.099 \\
\hline O 忧虑 & -.002 & .737 & -.231 & .053 & -.085 & .018 & -.146 & -.007 & -.244 \\
\hline Q1 实验 & -.030 & .037 & -.117 & -.110 & -.732 & -.205 & -.208 & .006 & .079 \\
\hline Q2 独立 & .032 & -.195 & -.245 & -.234 & .569 & -.149 & -.345 & -.090 & .015 \\
\hline Q3 自律 & .144 & -.031 & .789 & -.048 & .165 & -.136 & .210 & -.124 & .093 \\
\hline Q4 紧张 & -.115 & .787 & .029 & -.039 & .056 & -.005 & .171 & -.034 & -.094 \\
\hline A 乐群 & -.005 & .027 & -.006 & .779 & .044 & .086 & .108 & .136 & .099 \\
\hline $\begin{array}{c}\text { 一般认知的 } \\
\text { 表象 }\end{array}$ & .860 & -.052 & .085 & .004 & .113 & .072 & .014 & .072 & -.040 \\
\hline $\begin{array}{c}\text { 特殊认知的 } \\
\text { 表象 }\end{array}$ & .916 & .016 & .065 & -.032 & -.010 & .096 & .019 & -.013 & .029 \\
\hline $\begin{array}{c}\text { 激发控制动 } \\
\text { 机的表象 }\end{array}$ & .905 & .032 & .044 & .062 & $-7.093 \mathrm{E}-5$ & .091 & -.008 & -.022 & .015 \\
\hline $\begin{array}{c}\text { 激发唤醒表 } \\
\text { 象 }\end{array}$ & .833 & .038 & -.058 & .097 & -.020 & -.113 & .033 & .055 & .106 \\
\hline
\end{tabular}

如表 7 所示：根据因子得分系数矩阵可以得到各因子的得分函数：（F1=0.069 $\times$ 联慧 $+0.061 \times$ 稳定 $+0.104 \times$ 待强 $+0.203 \times$ 兴奋 $-0.041 \times$ 永恒 $+0.071 \times$ 敢为 $+0.073 \times$ 敏感 $+0.157 \times$ 怀疑 
$+0.053 \times$ 幻想 $+0.063 \times$ 事故 $-0.002 \times$ 忧虑 $-0.032 \times$ 独立 $+0.114 \times$ 自律 $-0.115 \times$ 紧张 $-0.005 \times$ 乐群 $+0.860 \times$ 一般认知的表象 $+0.916 \times$ 特殊认知的表象 $+0.905 \times$ 激发控制动机的表象 $+0.833 \times$ 激发唤醒动机的 表象）。从 F2 到 F9 的函数值的计算方法和 F1 算法相同。

\section{8 主成分因子指标回归分析}

\begin{tabular}{|c|c|c|c|c|c|c|}
\hline & & \multicolumn{2}{|c|}{ 未标准化系数 } & \multirow{3}{*}{$\begin{array}{c}\text { 标准化系数 } \\
\text { 标准误 }\end{array}$} & \multirow[t]{2}{*}{$\mathrm{t}$} & \multirow[t]{2}{*}{ Sig. } \\
\hline & & B & 标准误 & & & \\
\hline \multirow[t]{11}{*}{1} & (常数) & 54.889 & 5.346 & & 10.267 & .000 \\
\hline & $\mathrm{F} 1$ & 3.874 & 7.230 & .062 & .536 & .593 \\
\hline & $\mathrm{F} 2$ & 11.183 & 5.373 & .178 & 2.081 & $.039^{*}$ \\
\hline & F3 & 6.373 & 5.400 & .101 & 1.180 & .240 \\
\hline & F4 & -3.072 & 5.416 & -.049 & -.567 & .572 \\
\hline & F5 & 10.797 & 5.373 & .172 & 2.009 & $.047^{*}$ \\
\hline & F6 & -3.485 & 5.426 & -.055 & -.642 & .522 \\
\hline & F7 & -6.731 & 5.367 & -.107 & -1.254 & .212 \\
\hline & F8 & -4.762 & 5.371 & -.076 & -.887 & .377 \\
\hline & F9 & .877 & 5.391 & .014 & .163 & .871 \\
\hline & $\begin{array}{l}\text { 激发特殊动机 } \\
\text { 的表象 }\end{array}$ & -.271 & 7.369 & -.004 & -.037 & .971 \\
\hline & dent Variable: & & & & & \\
\hline
\end{tabular}

从表 8 中可以看出, 因子 F2 和 F5 都有显著性差异 $(\mathrm{P}<0.05)$ 标准误的系数最大, 其中 $\mathrm{F} 2$ 的值更显著, 而 F2 因子中的因素排名依次为 Q4 紧张性, O 忧虑性, $\mathrm{L}$ 怀疑性。而 F5 因子中的因素排名依次为 Q2 独立性、I 每感性和 B 聪慧性。

\section{4. 结论与建议}

4.1 优秀射箭运动员善于处理人际关系, 适应环境的能力较强, 情绪的成熟稳定、自律谨 慎，沉着，有自信心，激发控制动机的表象和特殊认知表象较高。

4.2 $16 \mathrm{PF}$ 中的心理因子对射箭成绩影响较大，因此，优秀射箭运动员的选材可采用 $16 \mathrm{PF}$ 人 格因素来测试。

4.3 优秀射箭运动员应选取低紧张性、心平气和、闲散宁静、低忧虑性、安祥沉着、自信心 强的选手作为参赛对象。

\section{致谢}

基金项目：2018 宁波市哲学社会科学学科带头人培育成果

\section{References}

[1] Lin Lin. Research on personality characteristics of elite Canoeing Athletes in China [J]. Journal of Shandong Institute of Physical Education and Sports 2010.3:27（3） : 48-50.

[2] Short SE, Bruggeman JM, Engel SG. The effect of imagery function and imagery direction on self-efficacy and performance on a golf-putting task[J].The Sport Psychologist, 2002, 16(1):48-67. 
[3] Cumming JL, Ste-Marie Dm. The cognitive and motivation effects of Imagery training[J].The Sport Psychologist, 2001,15(3):276-288. 